\title{
Study by Confocal Laser Scanning Microscopy of the Influence of Hydrogels on the Wound Healing Rate after Mechanical Corneal Injury
}

\author{
Pascal Furrer ${ }^{a \star}$, Frédéric Lallemand ${ }^{a}$, Grazia Maria Paladino ${ }^{b}$, Maria Grazia Mazzone ${ }^{b}$, \\ and Robert Gurny ${ }^{a}$
}

\begin{abstract}
Laser assisted refractive surgery is a common procedure for the correction of visual defects. However, this technique is sometimes associated with complications such as recurrent erosion scars or late-onset corneal haze formation due to irregular wound healing. In order to improve the risk/benefit ratio of this surgery, a better understanding of the wound healing process is absolutely necessary. The aim of the present study was to investigate in an animal model the influence of biopolymeric hydrogels in the modulation of the wound healing. Mechanical wounds were generated in rabbit cornea with the aid of an Algerbrush burr. The closure of the wound was monitored after staining with fluorescein by means of a confocal microscope. A hydrogel based on $1 \%$ xanthan gum stimulated significantly corneal wound healing whereas other viscosified solutions or hydrogels based on lower concentrations of xanthan gum or on hyaluronic acid did not promote wound healing.
\end{abstract}

Keywords: Cornea · Hydrogels · Ocular tolerance · Ophthalmic formulations · Refractive surgery

\section{Introduction}

Ametropic people have sought for a long time ways to improve their vision and rid themselves of eyeglasses. Surgical attempts at correcting vision by changing the anterior corneal curvature were crowned with success by the discovery of innovative techniques [1]. The most used techniques to reshape the cornea are photorefractive keratectomy (PRK) and laser-assisted in

${ }^{*}$ Correspondence: Dr. P. Furrera

Tel.: +412237933 36

Fax: +41223796567

E-Mail: Pascal.Furrer@pharm.unige.ch

aschool of Pharmaceutical Sciences

Ecole de Pharmacie Genève-Lausanne

Department of Pharmaceutics and Biopharmaceutics (FAGAL)

University of Geneva

Quai Ernest-Ansermet 30

$\mathrm{CH}-1211$ Geneva 4

bSIFI S.p.A. R\&D Department, I- 95020 Lavinaio

(Catania), Italy situ keratomileusis (LASIK). PRK treats ametropia with the aid of an argon fluoride excimer laser to reshape the anterior corneal stroma by photoablation after mechanically removing the epithelium. LASIK combines the creation of a hinged corneal flap using a microkeratome with the ablation of the exposed stromal bed using an excimer laser [2]. LASIK is the most popular refractive procedure as functional vision usually returns within one day whereas the functional vision recovery lasts 3-7 days with PRK. A relatively new laser surgical procedure, laser subepithelial keratomileusis (LASEK) combines certain elements of LASIK and PRK. The outcome of these surgical procedures is closely related to the wound-healing response [3]. Irregular healing is associated with recurrent erosions, subepithelial haze formation, postoperative pain or keratitis leading to slow visual rehabilitation [4]. Generally the corneal wound healing response is an exceedingly complex cascade involving both cytokine-mediated interactions between ocular tissues and cell events like keratocyte apoptosis, cell proliferation and migration, and inflammatory processes [5][6]. Numerous studies have been carried out to better understand the wound-healing process. Some authors used histologi- cal techniques for this purpose [7-9], others employed in vivo confocal microscopy to follow up the wound healing [10][11] The latter technique has the advantage of providing non invasive images of excellent quality in term of contrast and resolution without the artifacts induced by the preparation of the specimen in electron microscopy [10]. In order to promote the corneal epithelial regeneration after refractive surgery, some attempts have been made using mostly anti-inflammatory drugs or biopolymers like hyaluronic acid or chitosan [12][13]. Such polymers have the property to form hydrogels. These are defined as three-dimensional, hydrophilic, polymeric networks capable of imbibing large amounts of water [14]. They resemble natural living tissue more than any other synthetic biomaterials, due to their high water contents and soft consistency which is similar to natural tissue. They have been already used in ophthalmology to optimize ocular drug delivery of drug by prolonging the corneal contact time of the drug thus increasing ocular bioavailability [15]. In this context, the aim of the present study was to evaluate the effects that biopolymeric hydrogels can exert on the corneal reepithelization after mechanical injury in a clinically valid animal model. 


\section{Material and Methods}

\section{Animals and Treatment}

New Zealand white rabbits of both sexes and weighing approximately $2.5-3.5 \mathrm{~kg}$ (University Medical Center, Geneva, Switzerland) were used in this study. Animals were individually housed in stainless steel cages and maintained in a 12-h light/dark cycle at $19 \pm 1{ }^{\circ} \mathrm{C}$. They were allowed water and food ad libitum. All animals were healthy and free of clinically observable ocular abnormalities. All experiments were performed in accordance with the ARVO (Association for Research in Vision and Ophthalmology) statement for the use of animals in ophthalmic and vision research [16], and were approved by the local veterinary authority for animal experimentation.

Mechanical wounds in form of circular superficial epithelial abrasion were performed on rabbit corneas by means of an Algerbrush burr with a $1 \mathrm{~mm}$ tip (Jannach, Italy). Animals were anesthetized with an intramuscular injection of a 1:1 mixture of ketamine hydrochloride $(37.5 \mathrm{mg} / \mathrm{kg}$ body weight $)$ and xylazine hydrochloride $(10 \mathrm{mg} /$ $\mathrm{kg}$ bw). A drop of topical anesthetic (oxybuprocain hydrochloride $0.4 \%$, Novesin ${ }^{\circledR}$, Ciba Vision, Switzerland) was instilled on the cornea prior to the surgical procedure.

Circular wounds of $6 \mathrm{~mm}$ diameter were generated. The wound size was controlled by applying onto the cornea a sterilized transparent stencil film (Parafilm ${ }^{\circledR} \mathrm{M}$, American Can Company, Greenwich, USA) with a hole cut up by a punch of $6 \mathrm{~mm}$ diameter. Immediately after the creation of the mechanical wound, the rabbit eye was thoroughly rinsed by a sterile saline physiological solution (isotonic phosphate buffer solution).

Forty-eight rabbits were used in the study. Each rabbit received only one formulation without being reused for any further test. Each formulation was assessed on six animals. The hydrogel formulations were based on hyaluronic acid and xanthan gum. The compositions are summarized in the Table. The formulations were rendered isotonic to lachrymal fluid by addition of salts ( $\mathrm{NaCl}, \mathrm{KCl}, \mathrm{MgCl}_{2} 6 \mathrm{H}_{2} \mathrm{O}$, and $\mathrm{CaCl}_{2} 2 \mathrm{H}_{2} \mathrm{O}$ purchased from Fluka Chemie, Switzerland) and their $\mathrm{pH}$ was controlled by either phosphate buffer or tris (tris(hydroxymethy 1)aminomethane) buffer and adjusted to $\mathrm{pH}$ 7.2. They were viscosified by the addition of glycerol. The vehicle, a buffer saline solution served as control.

The different tested formulations were given on the right eye of the rabbits immediately after the surgical treatment at the beginning of the experiment. Then rabbits were treated with the different formulations three times per day at $9 \mathrm{~h}, 13 \mathrm{~h}$, and $17 \mathrm{~h}$ till the end of the experiment $(96 \mathrm{~h}$ after surgery).

Table. Composition of the different tested hydrogels and viscosified ophthalmic solutions

\begin{tabular}{|c|c|c|c|c|}
\hline Name of the product (abbreviation) & 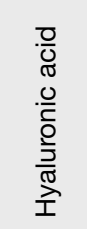 &  & $\begin{array}{l}\frac{1}{\Phi} \\
\frac{4}{5} \\
0 \\
0 \\
0 \\
\frac{1}{0} \\
\frac{c}{0} \\
\frac{0}{0} \\
0 \\
\frac{1}{0}\end{array}$ & 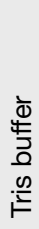 \\
\hline Eyestil ${ }^{\circledR}$ plus (eyestil) & $1.5 \%$ & - & $\mathrm{X}$ & \\
\hline EPG (EPG) & $1.5 \%$ & $1 \%$ & & $X$ \\
\hline Xanthan viscous solution (XNT 0.2\%) & - & $0.2 \%$ & & $\mathrm{X}$ \\
\hline Xanthan hydrogel (XNT 1\%) & - & $1 \%$ & & $X$ \\
\hline Vehicle (vehicle) & - & - & $X$ & \\
\hline
\end{tabular}

The abraded corneal surface was revealed by instilling a sterile isotonic sodium fluorescein solution $(0.5 \%, 25 \mu \mathrm{l})$. After 2 min dyeing, the excess fluorescein was washed out during one minute with a sterile $\mathrm{NaCl} 0.9 \%$ solution. The corneas were then observed by confocal microscopy.

\section{Microscopic Observation}

A confocal laser scanning ophthalmoscope (CLSO Zeiss, Oberkochen, Germany) modified by the addition of a set of lenses in order to view the cornea instead of the retina was used [17]. An argon ion laser operating at $488 \mathrm{~nm}$ wavelength was used as the excitation light source. The fluorescence signal was detected by a photomultiplier. Images were obtained using Epiplan-Neofluar 2.5×/0.075 NA objective lens (Zeiss, Oberkochen, Germany). Optical sectioning was performed parallel to the corneal surface, at 16 equidistant different focal planes, the focus shifting (from 0 to $470 \mu \mathrm{m}$ ) covering the whole corneal thickness. The images were displayed on a digital video monitor and recorded on a S-VHS videotape. An image processing system (Analysis SIS, Münster, Germany) carried out the following operation steps: addition of the 16 digitized images in a stack to produce a three-dimensional reconstruction, projection of this stack and calculation of the total surface of the fluorescent areas on the projection. The percentage of fluorescent areas on the observed corneal surface was compared for each animal to the initial wound surface $(100 \%)$. For observation, the rabbits were placed on a height-adjustable trolley in front of the camera head. Animals were evaluated for wound healing at different times: immediately after injury hour 0 , and further after 24 h, 48 h, 72 h, and 96 h. For these further (day 1 to 4 ) examinations, the animals were only sedated with an intramuscular injection $(0.25 \mathrm{ml} / \mathrm{kg}$ weight body) of medetomidine $0.1 \%$ (Domitor ${ }^{\circledR}$, Orion Corp., Espoo, Finland).

After examination, the sedative was antagonized with an intramuscular injection $(0.2 \mathrm{ml} / \mathrm{kg}$ weight body) of atipamezolin $\mathrm{HCl} 0.5 \%$ (Antisedan ${ }^{\circledR}$, Orion Corp., Espoo, Finland) and the rabbits were replaced in their cage.

\section{Statistical Analysis}

Results were evaluated in three ways. Firstly, the influence of the different treatments on the percentages of fluorescent corneal surface was compared to the vehicle treatment using the Student $\mathrm{t}$ test (unpaired sample, level of significance: $\mathrm{P}$ $>0.05)$ after a Fisher-Snedecor analysis of variance (supposing a normal distribution of the values) [18].

Secondly, using curve fitting in a form of a cubic spline (Maple 9 software, Maplesoft, Ontario, Canada), the mathematical equation of each curve (percentage of fluorescent area as a function of time) was calculated and the $\mathrm{t}_{20}$, i.e. the time needed to heal $80 \%$ of the wound, was determined [19-21].

Thirdly, using the same software, the Area Under the Curve (AUC) was determined for each curve (integration limits: $0 \mathrm{~h}$ and $96 \mathrm{~h}$ ). The AUC is a parameter indicating the healing rate: the smaller the AUC is, the faster the wound healing rate. A partial AUC was also calculated for bi-exponential curves (the interval of integration was taken from 0 hour until the time corresponding to the curve minimum point where the first derivative of the curve is zero) to take into account the first healing phase without the epithelial reorganization one.

\section{Results and Discussion}

The color reproductions (Fig. 1) of analyzed confocal images show the evolution 


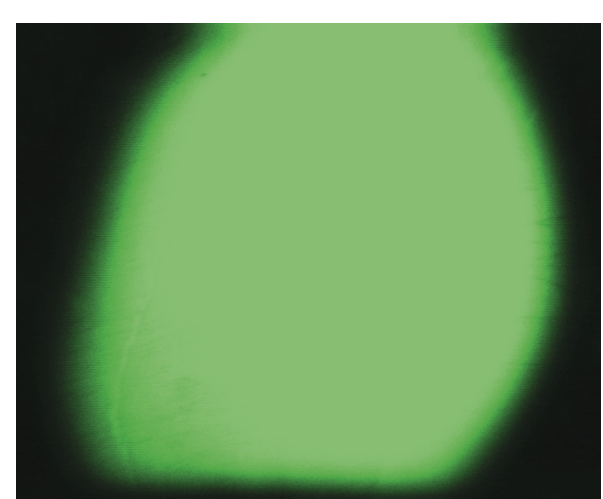

Fluorescent image of a rabbit cornea treated with the vehicle (saline solution), $24 \mathrm{~h}$ after surgery

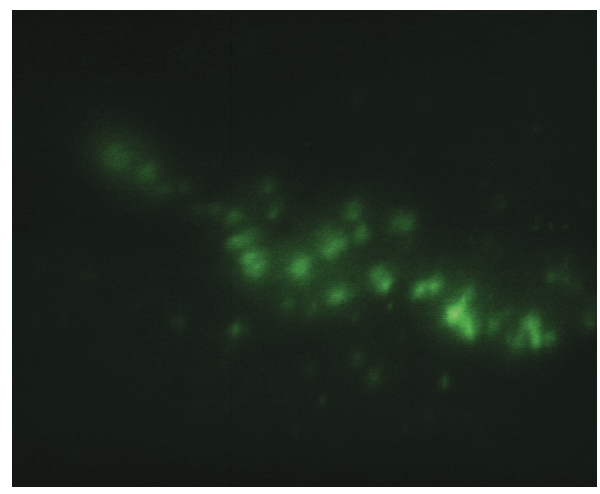

Fluorescent image of a rabbit cornea treated with the vehicle (saline solution), $72 \mathrm{~h}$ after surgery

Fig. 1.

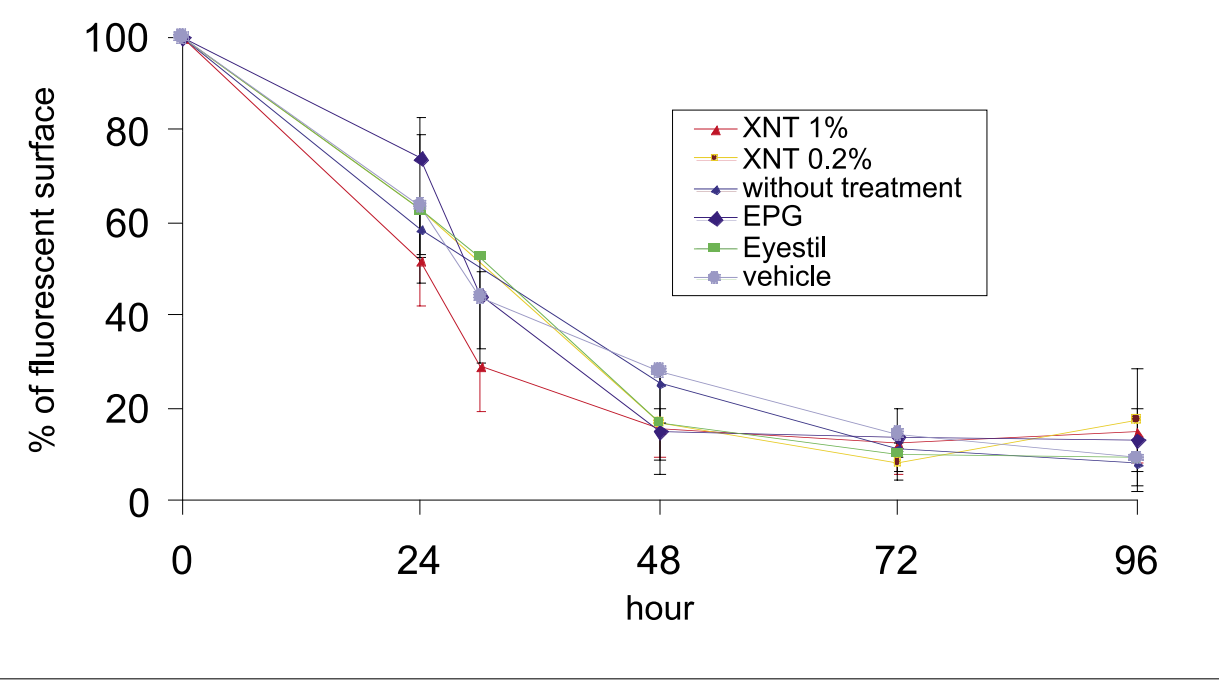

Fig. 2. Evolution of the rabbit corneal wound healing after circular mechanical wound of $6 \mathrm{~mm}$ diameter $(n=8$, mean $+\mathrm{SD})$. The effects on wound healing of two viscosified solutions (Eyestil and $0.2 \%$ xanthan gum - XNT $0.2 \%$ ) and two hydrogels (1\% xanthan gel - XNT $1 \%$ - and hyaluronic acid + xanthan gel-EPG) are compared with a saline solution (vehicle) and the absence of treatment (without treatment). The percentage of fluorescent corneal surface is assessed by confocal microscopy.

of the wound healing after treatment with the $1 \%$ xanthan gum hydrogel and the control saline solution.

These images show that the original rounded wound gets narrows with time; in every case, the wound is no longer visible after $72 \mathrm{~h}$. The wound is then completely



Fluorescent image of a rabbit cornea treated with the $1 \%$ xanthan gum hydrogel, $24 \mathrm{~h}$ after surgery

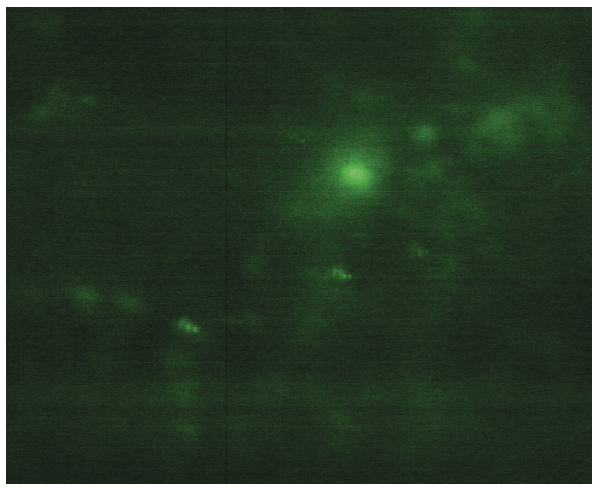

Fluorescent image of a rabbit cornea treated with the $1 \%$ xanthan gum hydrogel, $72 \mathrm{~h}$ after surgery closed up and only small spots appear indicating diffuse epithelial defects.

Fig. 2 shows the evolution of the wound healing after mechanical corneal injury for all tested products (mean $\pm \mathrm{SD}, \mathrm{n}=8$ ).

Globally, Fig. 2 shows a healing evolution that is very similar for all tested oph- thalmic formulations. This evolution consists of a rapid decrease of the percentage of fluorescent surface in $48 \mathrm{~h}$, followed by a slower decrease between $48 \mathrm{~h}$ and $96 \mathrm{~h}$. This evolution indicates a two-phase corneal repair mechanism. In the first phase, the wound closes up more or less rapidly, and in the second phase, a rearrangement of the epithelial cells occurs [22].

Due to unequal variance, the statistical comparison of the percentage of fluorescent wounded corneal area ( $\mathrm{t}$-test, $\mathrm{P}>0.05$, formulations versus saline solution) at different times $(24 \mathrm{~h}, 48 \mathrm{~h}, 72 \mathrm{~h}$, and $96 \mathrm{~h})$ does not discriminate the performance of the different formulations in terms of promoting wound healing. In order to assess eventual differences between the healing promoting effect of the tested formulations, two parameters are addressed: $\mathrm{t}_{20}$, i.e. the time needed to heal $80 \%$ of the wound, and partial AUC, i.e. the wound healing rate for the first corneal repair phase. For both parameters, $\mathrm{t}_{20}$ and $\mathrm{AUC}_{\text {partial }}$, the $1 \%$ xanthan gum hydrogel shows a significant promoting effect on wound healing rate compared to the saline blank solution. This effect does not appear for the other formulations. The comparison between both hydrogels, the $1 \%$ xanthan gum hydrogel and the combined formulation based on $0.15 \%$ hyaluronic acid and 1\% xanthan gel EPG, does not show any statistically significant difference, due to unequally large variance.

The results show that among the tested formulations, only the $1 \%$ xanthan gum hydrogel is seen to promote the wound healing as compared to the vehicle.

\section{Conclusion}

The present study has compared over 96 $\mathrm{h}$ the influence of hydrogels and viscosified ophthalmic formulations to a saline vehicle on the corneal epithelial healing after superficial mechanical wound.

The following conclusions can be drawn: as for the previous study, the in vivo confocal microscopic observation of the rabbit corneas has shown a two-phase wound healing process consisting of a rapid wound closure within $48 \mathrm{~h}$ followed by a slower wound healing and an epithelial reorganization. All tested formulations have shown this two-phase repair mechanism. Compared to the saline control solutions, only the hydrogel based on $1 \%$ xanthan gum has shown to stimulate significantly the corneal wound healing whereas other viscosified solutions or hydrogels have not been shown to promote wound healing. Surprisingly, the EPG hydrogel based on $0.15 \%$ hyaluronic acid and $1 \%$ xanthan gum was not more efficient than the $1 \%$ xanthan gum hydrogel. However due to large variance of 
the EPG-results, no statistically significant difference between both hydrogels can be concluded.

This study has shown the possible use of ophthalmic formulations to modulate the corneal wound healing rate after surgical injury. A further challenge would be to control the ocular inflammation caused by this injury.

\section{Acknowledgements}

The authors wish to thank Ms Catherine Siegfried for expert technical assistance during this study.

Received: April 12, 2005

[1] S. Taneri, J.D. Zieske, D.T Azar, Surv. Ophthalmol. 2004, 49, 576.

[2] M.C. Arbelaez, J.J. Pérez-Santonjy, M.M. Ismail, J.L. Alio, E. Chansue, J. Guell, R.J. Tsai, H.V. Gimbel, in 'Refractive Surgery, Current Techniques and Management', Ed. O.N.Serdarevic, Igaku-Shoin, New York, Tokyo, 1997, p. 131

[3] R.R. Mohan, A.E. Hutcheon, R. Choi, J. Hong, J. Lee, R.R. Mohan, R. Ambrosio, J.D. Zieske, S.E. Wilson, Exp. Eye Res. 2003, 76, 71.

[4] P. Fagerholm, J. Cataract Refract. Surg. 2000, 26, 432

[5] S.E. Wilson, R.R. Mohan, R.R. Mohan, R. Ambrosio, Jr., J. Hong, J. Lee, Prog. Retin. Eye Res. 2001, 20, 625.

[6] S.E. Wilson, M. Netto, R. Ambrosio, Jr., Am. J. Ophthalmol. 2003, 136, 530.

[7] I. Ratkay-Traub, B. Hopp, Z. Bor, L. Dux, D.L. Becker, T. Krenacs, Exp. Eye Res. 2001, 73, 291.

[8] T. Kato, K. Nakayasu, A. Kanai, Jpn. J. Ophthalmol. 2000, 44, 334.
[9] T. Miyamoto, S. Saika, A. Yamanaka, Y Kawashima, Y. Suzuki, Y. Ohnishi, J. Cataract Refract. Surg. 2003, 29,153.

[10] M.H. Vesaluoma, W.M. Petroll, J.J. PerezSantonja, T.U. Valle, J.L. Alio, T.M. Tervo, Am. J. Ophthalmol. 2000, 130, 564.

[11] T. Tervo, J. Moilanen, Prog. Retin. Eye Res. 2003, 22, 339.

[12] H. Ueno, T. Mori, T. Fujinaga, Adv. Drug Deliv. Rev. 2001, 52, 105

[13] E. Tani, C. Katakami, A. Negi, Jpn. J. Ophthalmol. 2002, 46, 488.

[14] N.A Peppas, P. Bures, W. Leobandung, H. Ichikawa, Eur. J. Pharm. Biopharm. 2000, 50, 27.

[15] O. Felt, S. Einmahl, P. Furrer, V. Baeyens, R. Gurny, in 'Polymeric Biomaterials', Ed. S. Dumitriu, Marcel Dekker, New York, 2002, p. 377.

[16] ARVO, Invest. Ophthalmol. Vis. Sci. 1994, 35 , v.

[17] P. Furrer, B. Plazonnet, J.M. Mayer, R. Gurny, Int. J. Pharm. 2000, 207, 89.

[18] J.E. De Muth, 'Basic Statistics and Pharmaceutical Statistical Applications', Marcel Dekker, New York, USA, 1999.

[19] J.H. Ahlberg, E.N. Nilson, J.L. Walsh, 'The Theory of Splines and Their Applications', Academic Press, New York, USA, 1967.

[20] C. de Boor, 'A Practical Guide to Splines', Springer-Verlag, Heidelberg, Germany, 1978.

[21] G.D. Knott, 'Interpolating Cubic Splines', Ed. U. Boston, Birkhäuser, Switzerland, 2000.

[22] C. Dohlman, Jpn. J. Ophthalmol. 1981, $25,131$. 